\title{
Non-invasive, Non-contact Heart Monitoring of Hemodialysis Patients with a Micropower Impulse Radar Technique
}

J. Chang, N. Levin, D. Poland, P. Welsh, C. Paulsen, J. Trebes, R. Rosenbury, T. Killip

\section{February 1, 2002}

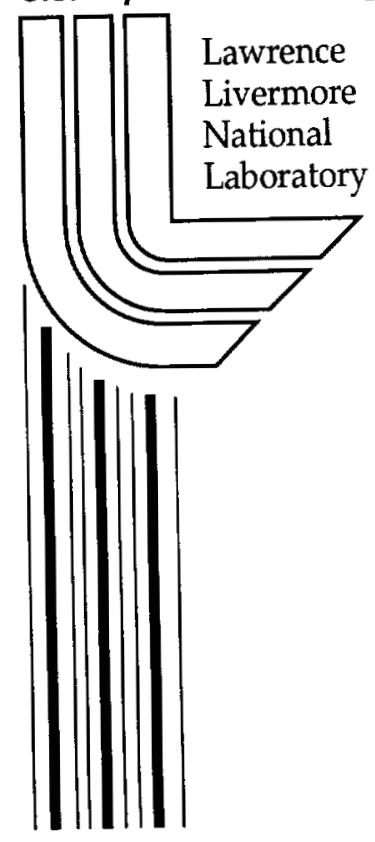




\section{DISCLAIMER}

This document was prepared as an account of work sponsored by an agency of the United States Government. Neither the United States Government nor the University of California nor any of their employees, makes any warranty, express or implied, or assumes any legal liability or responsibility for the accuracy, completeness, or usefulness of any information, apparatus, product, or process disclosed, or represents that its use would not infringe privately owned rights. Reference herein to any specific commercial product, process, or service by trade name, trademark, manufacturer, or otherwise, does not necessarily constitute or imply its endorsement, recommendation, or favoring by the United States Government or the University of California. The views and opinions of authors expressed herein do not necessarily state or reflect those of the United States Government or the University of California, and shall not be used for advertising or product endorsement purposes.

This work was performed under the auspices of the U. S. Department of Energy by the University of California, Lawrence Livermore National Laboratory under Contract No. W-7405-Eng-48.

This report has been reproduced directly from the best available copy.

Available electronically at http://www.doc.gov/bridge

Available for a processing fee to U.S. Department of Energy

And its contractors in paper from

U.S. Department of Energy

Office of Scientific and Technical Information

P.O. Box 62

Oak Ridge, TN 37831-0062

Telephone: (865) 576-8401

Facsimile: (865) 576-5728

E-mail: reports@adonis.osti.gov

Available for the sale to the public from

U.S. Department of Commerce

National Technical Information Service 5285 Port Royal Road

Springfield, VA 22161

Telephone: (800) 553-6847

Facsimile: (703) 605-6900

E-mail: orders@ntis.fedworld.gov

Online ordering: http://www.ntis.gov/ordering.htm

\section{OR}

Lawrence Livermore National Laboratory

Technical Information Department's Digital Library

http://www.llnl.gov/tid/Library.html 


\title{
Non-invasive, Non-contact Heart Monitoring of Hemodialysis Patients with a Micropower Impulse Radar Technique
}

\author{
J. Chang*, N. Levin**, D. Poland*, P. Welsh*, C. Paulsen*, J. Trebes*, R. Rosenbury*, \\ T. Killip*** \\ *Lawrence Livermore National Laboratory \\ **Renal Research Institute \\ ***Beth Israel Medical Center
}

\begin{abstract}
This report summarizes the LLNL LDRD funded portion of a collaborative project to demonstrate and clinically evaluate the micropower impulse radar technology as a means to non-invasively monitor the heart of chronic care patients undergoing hemodialysis. The development is based upon technologies and expertise unique to LLNL. The LLNL LDRD funded portion of this project was used to assist in the definition, design, construction, and evaluation of the prototype.
\end{abstract}

\section{Introduction}

Kidney dialysis patients frequently have heart and vascular problems. Frequent monitoring of the heart could lead to early detection and treatment of these problems as well as providing some of the basic data necessary for understanding the development and progress of these health problems. A non -invasive, non -contact method of monitoring the heart during hemodialysis could provide the opportunity for facilitating this type of monitoring without the discomfort of EKG connections or the intrusion of a doctor and stethoscope. Current monitoring techniques are intrusive, uncomfortable, and labor intensive, especially considering that hemodialysis is performed on each patient in a dialysis center 3 times a week for 4 hours each time. Furthermore, this radar technique can be beneficial in that it is sensing the actual heart motion and not just the electrical signals of the heart.

Atrial and ventricular cardiac arrhythmias and sudden death are the leading cause of death during dialysis, accounting for $80 \%$ of all sudden deaths in patients receiving hemodialysis. Main causes of cardiac arrhythmias are thought to be presence of coronary artery disease and left ventricular hypertrophy associated with one or several contributory factors such as intradialytic hemodynamic changes and fluctuations in electrolytes and pH. (1)

Continuous monitoring of heart activity during dialysis allowing for early detection and treatment of adverse cardiac events is of paramount importance in prevention of sudden death particularly in high risk patients with poor myocardial function and coronary disease. An established and time-tested method to monitor heart function is detection and recording of electric potentials generated by the conductive system of the heart, electrocardiogram or ECG, requires sophisticated electronic equipment and temporary 
attachment of number of recording electrodes to the patient's body. The cost of the equipment and the time required for proper placement of the recording electrodes multiplied by the sizeable number of patients on dialysis make this method impractical for general use. An inexpensive device, particularly one that could be integrated with the dialysis equipment and capable of detecting the heart activity without requiring direct contact with the patient body would be highly desirable, particularly if the method were simple.

The objective of the overall study is to determine if the micropower impulse radar device can be used with a high degree of sensitivity and specificity to detect the presence of cardiac arrhythmia. The system will be based on LLNL developed prototype systems that have already been demonstrated on Institutional Review Board (IRB) approved human subjects. $(2,3,4)$ The emitted electromagnetic radiation has both peak and average power levels that are orders of magnitude lower than that of a hand-held cellular phone and well below the level of existing safety standards for non-ionizing radiation, and is thus believe to be of no health threat. The impulse radar technology generates and detects extremely low-power ultra-wideband electromagnetic pulses in the Gigahertz regime in a compact portable configuration. The key technical question to be addressed in this project is whether it will be possible to detect and monitor cardiac arrhythmia in patients undergoing hemodialysis.

The aim of this portion of the feasibility study (funded by the LDRD) is to design and build a Micropower Impulse Radar (MIR) prototype to specifically monitor heart activity and possibly detect serious rhythm disturbances and hemodynamic changes in a patient on hemodialysis. While the end goal of the overall project is to study hemodialysis care patients, IRB approved healthy human subjects will be recruited for the initial study. The prototype radar system will be based upon a current LLNL radar design with a slight modification in signal generating characteristics more conducive for human body interrogation. The LDRD grant was used in part to fund the fabrication of the radar designed to measure cardiac motion of human subjects with varying body types and sizes. Clinical studies (to be funded by the Renal Research Institute) will evaluate the prototype against ECG readings of healthy volunteers recruited at the Yorkville Dialysis Center in New York by Renal Research Institute, Beth Israel Medical Center, and/or Mount Sinai Medical Center personnel.

20 healthy volunteers demographically representing ages $18-75$, including women and minorities will be recruited. ECG leads will be attached to the volunteer. The ECG data will verify that the MIR signal is indeed the heart rate. The ECG waveform and MIR signal will be captured and stored on a laptop.

Expected clinical data will subsequently be used to evaluate the basic utility of a radar based cardiac monitor for chronic care monitoring. The data analysis and ECG/radar signature comparison will be conducted at both LLNL and RRI. The determination of the fidelity and utility of the radar device as a cardiac monitor will also be evaluated by the participating cardiologist. Upon a successful completion of studying healthy volunteers, a new protocol will be proposed to investigate ECG and radar responses of actual 
hemodialysis patients.

Benefits resulting from this study has the potential of providing chronic hemodialysis patients with improved quality of care during the dialysis procedure and possibly anticipate and prevent cardiac disease as a result of the effects of long term hemodialysis treatments.

This project was performed under the mission direction to support the LLNL Physics and Advance Technology Directorate's Medical Technology Program (MTP). External collaboration includes Dr. Nathan Levin, Medical and Research Director of the Renal Research Institute and Dr. Thomas Killip, Director of the Killip Heart Institute at the Beth Israel Medical Center.

\section{Results}

The funds received from LDRD in FY01 for 6 months of exploratory funding resulted in the following achievements.

1. We have determined the basic system requirements of the prototype and needs of the clinician. We have specifically focus on the detection of the cardiac rhythm of individuals of various body types as illuminated from the front and the back of an individual. Previous observations of radar prototypes with a center frequencies above $2 \mathrm{GHz}$ have shown that ability to detect cardiac rhythm is dependent significantly upon the center frequency of operation and antenna design. It appeared that the lower the center frequency of operation, the more effective it was to measure higher weight individuals. This might be due in part to the effect of thick chest walls in heavier individual where signal attenuation becomes greater. Existing prototypes operating at a center frequency of approximately $2 \mathrm{GHz}$ was capable of monitoring individuals only below approximately $150 \mathrm{lbs}$. Because human body sizes can vary significantly greater than $150 \mathrm{lb}$, it was decided that a prototype radar needed to be fabricated with a lower center of frequency $(900 \mathrm{MHz})$ such that signal penetration can be enhanced for individuals with thicker thoracic walls. In this manner, it will be possible to leverage current MIR design with minimal hardware changes and matched to available funding.

2. We have used LLNL electromagnetic 3D Visible Man modeling capabilities (expertise in 3D FDTD modeling using commercially available computational package) and LLNL collected dielectric data to design the prototype radar. We leveraged current and prior investigations (expertise unique to LLNL) into potential medical applications of the micropower impulse radar technique such as the detection of intracranial hematoma and pneumothorax. Figure 1 to Figure 3 show examples of the computational modeling performed in an attempt to better understand the behavior of pulsed electromagnetic signals in the human thorax and illuminated from the rear. The images show the sagittal cross section of a human male FDTD descretized with $5 \mathrm{~mm}$ cubical cells. Each figure shows the scattered E-field magnitude distribution without and with the underlying human thorax mesh 
distribution. The color in the mesh is coded for different tissue properties. The incident pulse used for the study was 300ps. Figures 1 and 2 shows the field distribution 3.3ns after incident pulse generation from the center of the back. Figures 1 and 2 also shows the resulting changes in the field distribution when an antenna reflector is varied from straight to curved. This capability allows us a means to predict and pre-evaluate different antenna configurations. Figures 2 and 3 shows the E-field distribution at $3.3 \mathrm{~ns}$ and $5.4 \mathrm{~ns}$, respectively, after incident impulse. Note the significant effect of the scattered fields due to the presence of various organs in the thoracic cavity.

3. One prototype radar device was constructed and underwent preliminary evaluation. Figure 4 is a picture of the device. These evaluation included bench top analysis of device performance in terms of transceiver dynamic range and range sensitivity. The device composes of two parts, the antenna (right) and the transceiver (boxed). This device was used to as the front end of the system to develop the user interfaces described below.

4. Labview based user/computer interfaces with real time display and real time signal processing of radar signatures were written. These software facilitated data acquisition from the MIR onto laptop computers for efficient signal processing. The signals are displayed in time domain and frequency domain (FFT and low pass filtering).

5. Preliminary evaluation of the prototype sensitivity to varying body sizes and illumination orientation have shown encouraging results. This observation is estimated based upon simulating signal sensitivity on in-animate targets such as chair backs with different obstacles as absorbing and scattering medium.

6. A human subject studies protocol has been developed and is currently awaiting final approval by the Beth Israel Medical Center and LLNL Institutional Review Board. Because of personnel changes at RRI and resulting delay in the processing of the IRB review, we were unable to proceed with the human subjects studies during FY01. We anticipate human subjects studies to begin in FY02 upon IRB approval.

7. Since the prototype was specifically designed to monitor heart rhythm and is sensitive to motion in general, the device was slightly modified and tuned for use as a debris penetrating radar to search for live victims that might have been trapped in voided regions in building debris. This was done in responding to FEMA and DOE request to assist with the search and rescue efforts at World Trade Center from the September 11,2001 terrorist bombing. In addition, Dr. Chang, as a member of a 10 member team from LLNL, responded to the scene and assisted with deployment of similar devices.

\section{Plans for FY02}

Patients requiring regular hemodialysis as well as normal healthy individuals will be selected and undergo cardiac monitoring with the micropower impulse radar detector at the Beth Israel Medical Center. This portion of the study will be funded by the Renal Research Institute. 
Involvement of Human Subjects (expected clinical study)

The MIR device will be mounted onto the back of a dialysis chair with the signal emitting aperture directed through the back of the chair. No special preparation of the patient will be necessary. The investigator(s) will record signals collected from the MIR onto a portable computer. The cardiac monitoring period will vary between a few minutes to approximately 4 hours, the time necessary to perform the hemodialysis. Exact period of monitoring will be chosen at the discretion of the attending physician. The recorded signals will be benchmarked against simultaneously recorded EKG traces.

\section{Conclusion}

The results to date have suggested that the tools and the techniques have been obtained that will allow us to proceed into clinical studies of both healthy and chronically ill human subjects for the monitoring of cardiac arrhythmia using the micropower impulse radar technology.

\section{Acknowledgement}

Additional contributions have been made by Mary Carter from the Renal Research Institute. 


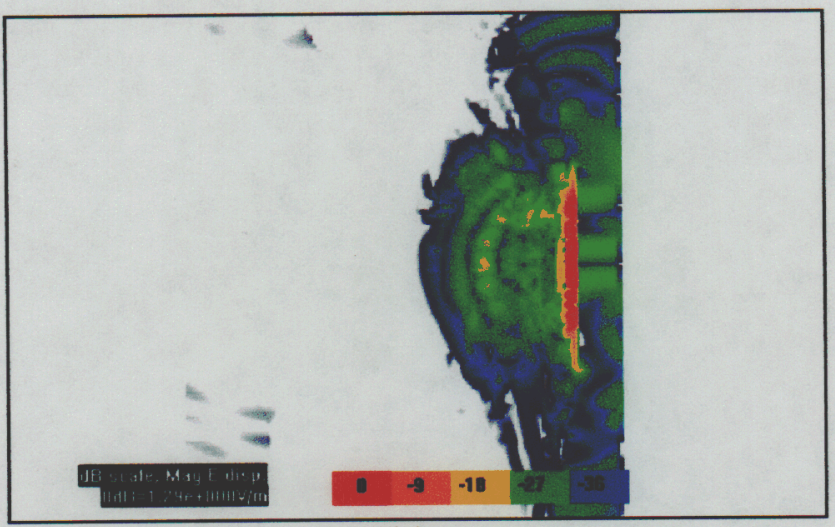

(a)

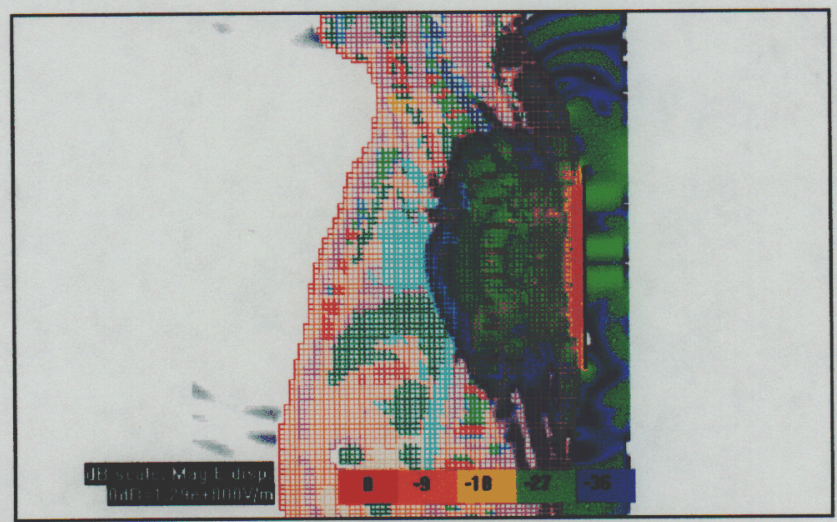

(b)

Figure 1 Sagittal cross section of the impulse field distribution of a human male thorax (a) without descritizing mesh visible and (b) with discretizing mesh visible. Straight backed antenna reflector. 

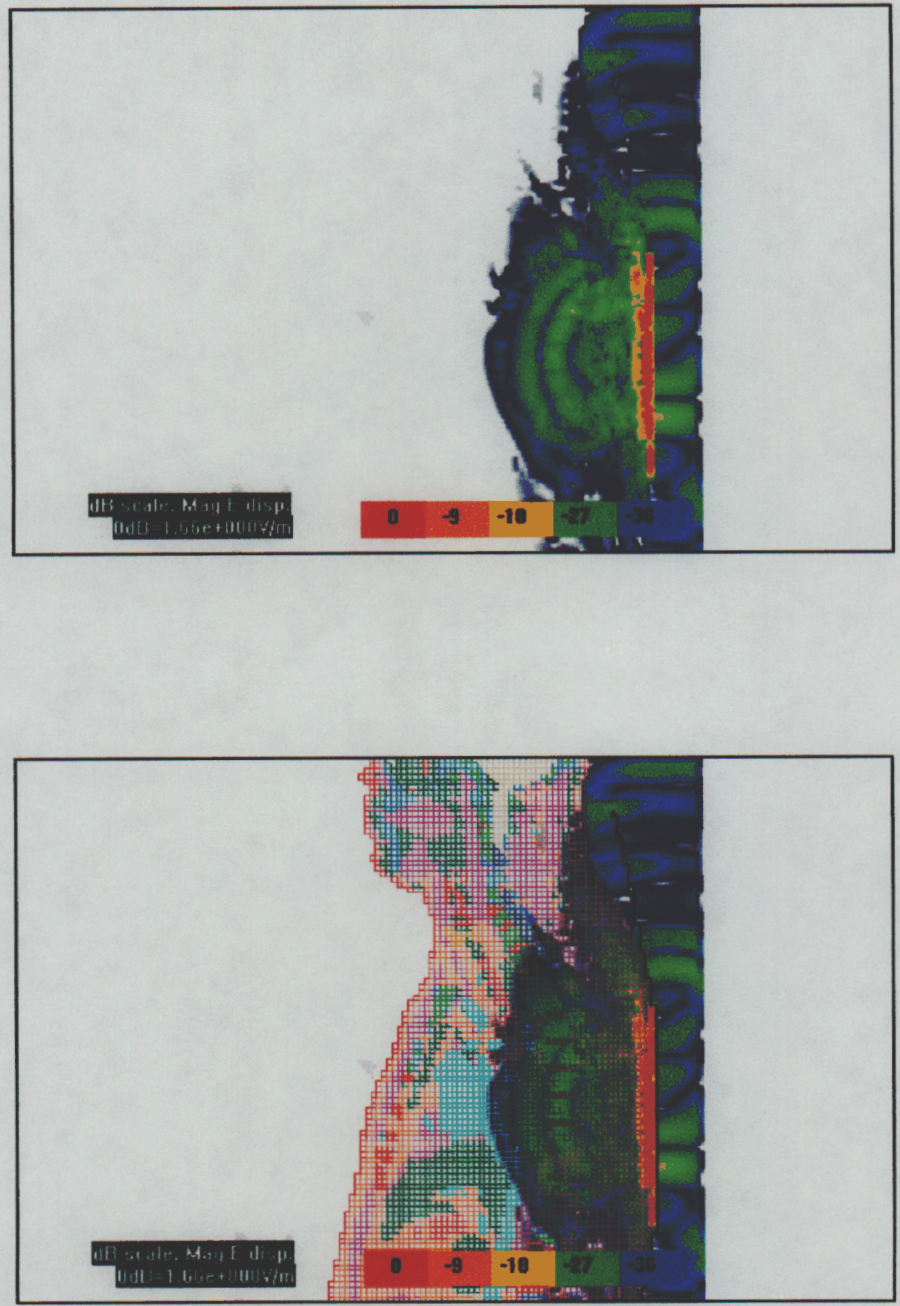

Figure 2 Sagittal cross section of the impulse field distribution of a human male thorax (a) without descritizing mesh visible and (b) with discretizing mesh visible. Curved backed antenna reflector. $3.3 \mathrm{~ns}$ after incident pulse. 

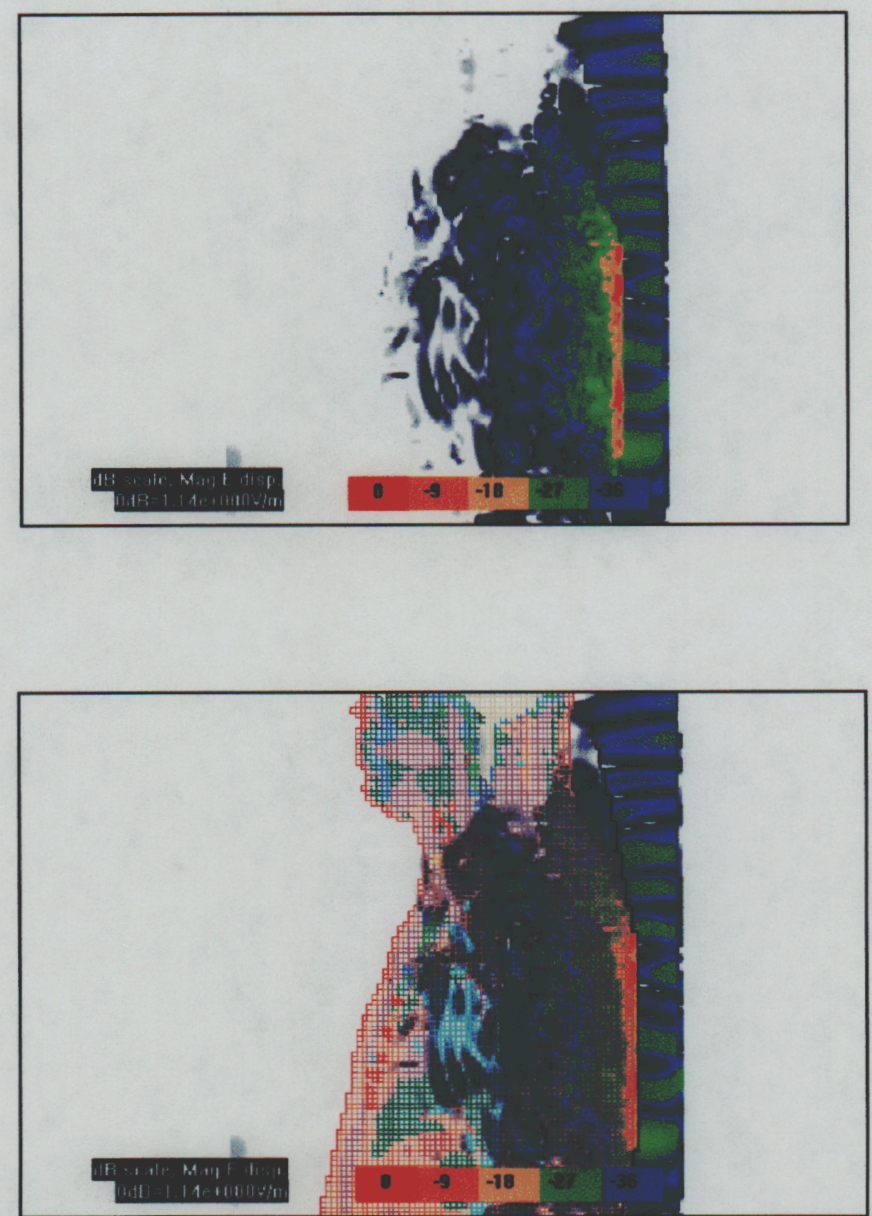

Figure 3 Sagittal cross section of the impulse field distribution of a human male thorax (a) without descritizing mesh visible and (b) with discretizing mesh visible. Curved backed antenna reflector. $5.4 \mathrm{~ns}$ after incident pulse. 


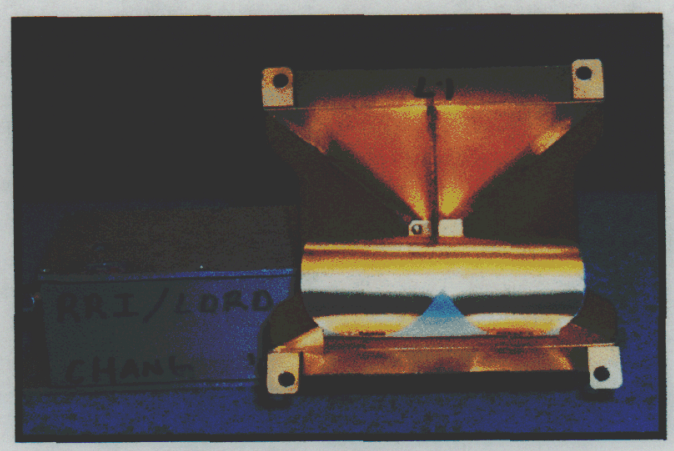

Figure 4. Radar prototype with attached antenna.

\section{Reference}

1. Chazan J. Sudden Death in Patients with Chronic Renal Failure on Hemodialysis. $3^{\text {rd }}$ Edition, Edited by Nissenson AR, Fine RN, Gentile DE. Appleton and Lange, 1995; 235-267.

2. Azevedo SG, McEwan TE. Micropower Impulse Radar, Science and Technology Review, UCRL-52000-96-1/2, January 1996.

3. Mast JE, Azevedo SG, Haddad W, Ng L, Burnett G. Micropwer Impulse Radar Technology and Applications, Lawrence Livermore National Laboratory, UCRL-ID130474, April 15, 1998.

4. Haddad W, Chang J, Rosenbury T, Dallum G, Welsh P, Scott D, Duarte D, AcevedoBolton V. Microwave Hematoma Detector for the Rapid Assessment of Head Injuries, Lawrence Livermore National Laboratory, UCRL-ID-137901, February 11, 2000. 